\title{
Quasiopen and $p$-Path Open Sets, and Characterizations of Quasicontinuity
}

\author{
Anders Björn' ${ }^{1}$ Jana Björn' ${ }^{1} \cdot J_{a n}$ Malý $^{2}$
}

Received: 9 September 2015 / Accepted: 30 July 2016 / Published online: 1 September 2016

(C) The Author(s) 2016. This article is published with open access at Springerlink.com

\begin{abstract}
In this paper we give various characterizations of quasiopen sets and quasicontinuous functions on metric spaces. For complete metric spaces equipped with a doubling measure supporting a $p$-Poincaré inequality we show that quasiopen and $p$-path open sets coincide. Under the same assumptions we show that all Newton-Sobolev functions on quasiopen sets are quasicontinuous.
\end{abstract}

Keywords Analytic set - Characterization - Doubling measure - Fine potential theory · Metric space $\cdot$ Newtonian space $\cdot$ Nonlinear potential theory $\cdot$ Poincaré inequality $\cdot p$-path open · Quasicontinuous · Quasiopen · Sobolev space · Suslin set

Mathematics Subject Classification (2010) Primary: 31E05; Secondary: 28A05 . $30 \mathrm{~L} 99 \cdot 31 \mathrm{C} 15 \cdot 31 \mathrm{C} 40 \cdot 31 \mathrm{C} 45 \cdot 46 \mathrm{E} 35$

\section{Introduction}

When studying Sobolev spaces and potential theory on an open subset $\Omega$ of $\mathbf{R}^{n}$ (or of a metric space), there are two natural Sobolev capacities one can consider. One defined using

Jana Björn

jana.bjorn@liu.se

Anders Björn

anders.bjorn@liu.se

Jan Malý

maly@karlin.mff.cuni.cz

1 Department of Mathematics, Linköping University,

SE-581 83 Linköping, Sweden

2 Department of Mathematics, Faculty of Science, J. E. Purkyně University,

České mládeže 8, CZ-400 96 Ústí nad Labem, Czech Republic 
the global Sobolev norm and the other one using the Sobolev norm on $\Omega$. When $\Omega$ is open, these two capacities are easily shown to have the same zero sets. We shall show that the same holds also if $\Omega$ is only quasiopen, i.e. open up to sets of arbitrarily small capacity, see Proposition 4.2 for the exact details.

To consider Sobolev spaces and capacities on nonopen sets is natural e.g. in fine potential theory. Such studies were pursued on quasiopen sets in $\mathbf{R}^{n}$ by KilpeläinenMalý [22], Latvala [24] and Malý-Ziemer [25]. In the last two decades, several types of Sobolev spaces have been introduced on general metric spaces by e.g. Cheeger [11], Hajłasz [14] and Shanmugalingam [28]. Using this approach one just regards subsets as metric spaces in their own right, with the metric and the measure inherited from the underlying space. This makes it possible to define Sobolev type spaces on even more general subsets.

We shall use the Newtonian Sobolev spaces, which on open subsets of $\mathbf{R}^{n}$ are known to coincide with the usual Sobolev spaces, see Theorem 4.5 in Shanmugalingam [28] and Theorem 7.13 in Hajłasz [14]. This equivalence is true also for open subsets of weighted $\mathbf{R}^{n}$ with $p$-admissible weights, $p>1$, see Propositions A.12 and A.13 in [4]. See also Heinonen-Koskela-Shanmugalingam-Tyson [19] for more on Newtonian spaces.

It is well known that every equivalence class of the classical Sobolev spaces contains better-than-usual, so-called quasicontinuous, representatives. In metric spaces this is only known to hold under certain assumptions. Roughly speaking, quasicontinuity means continuity outside sets of arbitrarily small capacity, see Definition 3.1. The Sobolev capacity plays a central role when defining quasicontinuity, and there are actually two types of quasicontinuity that one can consider on $\Omega$, one for each of the two capacities mentioned above. As far as we know this subtle distinction has not been discussed in the literature. It is not difficult to show that these two notions of quasicontinuity are equivalent if $\Omega$ is open, and examples show that for general sets this is not true.

We shall show in Proposition 3.4 that the equivalence holds for functions defined on quasiopen sets. The proof is more involved than for open sets, but still rather elementary, and it holds in arbitrary metric spaces (only assuming that balls have finite measure). In Proposition 3.3 we obtain a similar equivalence for two notions of quasiopenness. These two results (and also Proposition 4.2) complement the restriction result from Björn-Björn [5, Proposition 3.5], stating that if $U \subset X$ is $p$-path open and measurable, then the minimal $p$-weak upper gradients with respect to $X$ and $U$ coincide in $U$. All these results show the equivalence between a global property and the corresponding property localized to a quasiopen or $p$-path open set.

It was shown by Shanmugalingam [29, Remark 3.5] that quasiopen sets in arbitrary metric spaces are $p$-path open, i.e. that $p$-almost every rectifiable curve meets such a set in a relatively open 1-dimensional set. We shall show that under the usual assumptions on the metric space (and in particular in $\mathbf{R}^{n}$ ), the converse implication is true as well. More precisely, we prove the following result.

Theorem 1.1 Assume that the metric space $X$ equipped with a doubling measure $\mu$ is complete and supports a p-Poincaré inequality. Then every p-path open set in $X$ is quasiopen (and in particular measurable).

Under the same assumptions it was recently shown by Björn-Björn-Latvala [8, Theorem 1.4] that a set is quasiopen if and only if it is a union of a finely open set and a set with zero capacity, generalizing a similar result from $\mathbf{R}^{n}$, see Adams-Lewis [1, Proposition 3]. Thus we now have two characterizations of quasiopen sets. 
In Example 4.7 we show that in more general metric spaces it can happen that not all $p$-path open sets are measurable, let alone quasiopen.

As a consequence of Theorem 1.1 we obtain the following characterization of quasicontinuous functions.

Theorem 1.2 Assume that the metric space $X$ equipped with a doubling measure $\mu$ is complete and supports a p-Poincaré inequality. Let $U \subset X$ be quasiopen.

Then $u: U \rightarrow[-\infty, \infty]$ is quasicontinuous if and only if it is measurable and finite q.e., and $u \circ \gamma$ is continuous for $p$-a.e. curve $\gamma:\left[0, l_{\gamma}\right] \rightarrow U$.

In Proposition 3.4, we also provide a characterization of quasicontinuity using quasiopen sets in the spirit of Fuglede [13, Lemma 3.3]. In [8, Theorem 1.4] yet another characterization of quasicontinuity was given, this time in terms of fine continuity, see also [13, Lemma, p. 143].

Newtonian functions are defined more precisely than the usual Sobolev functions (in the sense that the classes of representatives are narrower), and under the assumptions of Theorem 1.1 it was shown in Björn-Björn-Shanmugalingam [10] that all Newtonian functions on $X$ and on open subsets of $X$ are quasicontinuous. Moreover, the recent results in Ambrosio-Colombo-Di Marino [2] and Ambrosio-Gigli-Savaré [3] imply that the same holds if $X$ is a complete doubling metric space and $1<p<\infty$.

Using the characterization in Theorem 1.2 we can extend the quasicontinuity result from [10] to quasiopen sets as follows. See also Björn-Björn-Latvala [7] and Remark 4.6.

Theorem 1.3 Assume that the metric space $X$ equipped with a doubling measure $\mu$ is complete and supports a p-Poincaré inequality. Let $U \subset X$ be quasiopen. Then every function $u \in N_{\mathrm{loc}}^{1, p}(U)$ is quasicontinuous.

In the last section we weaken the assumptions in Theorem 1.1 and replace the doubling property and the Poincare inequality by the requirement that bounded Newtonian functions are quasicontinuous, which is a much weaker assumption. In particular, we obtain the following result. In Section 5 we give a more general version using coanalytic sets.

Theorem 1.4 Assume that $X$ is complete, and that every bounded $u \in N^{1, p}(X)$ is quasicontinuous. Then every Borel p-path open set $U \subset X$ is quasiopen.

We also prove a similar modification of Theorem 1.2, see Proposition 5.1. Our proofs of these generalized results (without doubling and Poincaré assumptions) are based on the following result which guarantees measurability of certain functions defined by their upper gradients. It may be of independent interest, and generalizes Corollary 1.10 from Järvenpää-Järvenpää-Rogovin-Rogovin-Shanmugalingam [20], where a similar measurability result was proved in the singleton case $X \backslash U=\left\{x_{0}\right\}$.

Proposition 1.5 Assume that $X$ is complete and separable, and that $U$ is a coanalytic set. For every Borel function $\rho: X \rightarrow[0, \infty]$ define

$$
u_{\rho}(x)=\inf _{\gamma \in \Gamma_{x}} \int_{\gamma} \rho d s
$$


where $\Gamma_{x}$ is the family of all rectifiable curves $\gamma:\left[0, l_{\gamma}\right] \rightarrow X$ (including constant curves) such that $\gamma(0)=x$ and $\gamma\left(l_{\gamma}\right) \in X \backslash U$. Then $u_{\rho}$ is measurable.

The paper is organized as follows. In Section 2 we recall the necessary background on Newtonian spaces. Section 3 deals with the two notions of quasicontinuity. The results in that section are valid in arbitrary metric spaces. Theorems 1.1-1.3 are proved in Section 4. In Section 5 some partial generalizations of the results from Section 4 are proved without the Poincaré and doubling assumptions. We also formulate two open problems about Borel representatives of Newtonian functions.

\section{Notation and Preliminaries}

We assume throughout this paper that $1 \leq p<\infty$ and that $X=(X, d, \mu)$ is a metric space equipped with a metric $d$ and a positive complete Borel measure $\mu$ such that $\mu(B)<\infty$ for all open balls $B \subset X$.

A curve is a continuous mapping from an interval, and a rectifiable curve $\gamma$ is a curve with finite length $l_{\gamma}$. We will only consider curves which are compact and rectifiable. Unless otherwise stated they will also be nonconstant and parameterized by arc length $d s$.

For a family of curves $\Gamma$ on $X$, we define its $p$-modulus

$$
\operatorname{Mod}_{p}(\Gamma):=\inf \int_{X} \rho^{p} d \mu,
$$

where the infimum is taken over all nonnegative Borel functions $\rho$ such that $\int_{\gamma} \rho d s \geq 1$ for all $\gamma \in \Gamma$. A property is said to hold for $p$-almost every curve if it fails only for a curve family $\Gamma$ with zero $p$-modulus. Following Heinonen-Koskela [18], we introduce upper gradients as follows (they called them very weak gradients).

Definition 2.1 A nonnegative Borel function $g$ on $X$ is an upper gradient of an extended real-valued function $f$ on $X$ if for all nonconstant, compact and rectifiable curves $\gamma$ : $\left[0, l_{\gamma}\right] \rightarrow X$

$$
\left|f(\gamma(0))-f\left(\gamma\left(l_{\gamma}\right)\right)\right| \leq \int_{\gamma} g d s,
$$

where we follow the convention that the left-hand side is $\infty$ whenever at least one of the terms therein is infinite. If $g$ is a nonnegative measurable function on $X$ and if Eq. 2.1 holds for $p$-almost every curve, then $g$ is a $p$-weak upper gradient of $f$.

Note that a $p$-weak upper gradient need not be a Borel function, it is only required to be measurable. The $p$-weak upper gradients were introduced in Koskela-MacManus [23]. It was also shown there that if $g \in L^{p}(X)$ is a $p$-weak upper gradient of $f$, then one can find a sequence $\left\{g_{j}\right\}_{j=1}^{\infty}$ of upper gradients of $f$ such that $g_{j} \rightarrow g$ in $L^{p}(X)$. If $f$ has an upper gradient in $L^{p}(X)$, then it has a minimal p-weak upper gradient $g_{f} \in L^{p}(X)$ in the sense that for every $p$-weak upper gradient $g \in L^{p}(X)$ of $f$ we have $g_{f} \leq g$ a.e., see Shanmugalingam [29] and Hajłasz [14]. The minimal $p$-weak upper gradient is well defined up to a set of measure zero in the cone of nonnegative functions in $L^{p}(X)$. Following Shanmugalingam [28], we define a version of Sobolev spaces on the metric measure space $X$. 
Definition 2.2 Let for measurable $f$,

$$
\|f\|_{N^{1, p}(X)}=\left(\int_{X}|f|^{p} d \mu+\inf _{g} \int_{X} g^{p} d \mu\right)^{1 / p},
$$

where the infimum is taken over all upper gradients $g$ of $f$. The Newtonian space on $X$ is

$$
N^{1, p}(X)=\left\{f:\|f\|_{N^{1, p}(X)}<\infty\right\} .
$$

The space $N^{1, p}(X) / \sim$, where $f \sim h$ if and only if $\|f-h\|_{N^{1, p}(X)}=0$, is a Banach space and a lattice, see Shanmugalingam [28]. In this paper we assume that functions in $N^{1, p}(X)$ are defined everywhere, not just up to an equivalence class in the corresponding function space. Nevertheless, we will still say that $\tilde{u}$ is a representative of $u$ if $\tilde{u} \sim u$.

Definition 2.3 The Sobolev capacity of an arbitrary set $E \subset X$ is

$$
C_{p}(E)=\inf _{u}\|u\|_{N^{1, p}(X)}^{p},
$$

where the infimum is taken over all $u \in N^{1, p}(X)$ such that $u \geq 1$ on $E$.

The capacity is countably subadditive. A property holds quasieverywhere (q.e.) if the set of points for which the property does not hold has capacity zero. The capacity is the correct gauge for distinguishing between two Newtonian functions. If $u \in N^{1, p}(X)$ and $v$ is everywhere defined, then $v \sim u$ if and only if $v=u$ q.e. Moreover, Corollary 3.3 in Shanmugalingam [28] shows that if $u, v \in N^{1, p}(X)$ and $u=v$ a.e., then $u=v$ q.e. In particular, $\tilde{u}$ is a representative of $u$ if and only if $\tilde{u}=u$ q.e.

We thus see that the equivalence classes in $N^{1, p}(X) / \sim$ are more narrowly defined than for the usual Sobolev spaces. In weighted $\mathbf{R}^{n}$ (with a $p$-admissible weight and $p>1), N^{1, p}\left(\mathbf{R}^{n}\right) / \sim$ coincides with the refined Sobolev space as defined in HeinonenKilpeläinen-Martio [17, p. 96], see Björn-Björn [4, Appendix A.2] (or [28] and BjörnBjörn-Shanmugalingam [10] for unweighted $\mathbf{R}^{n}$ ).

For a measurable set $U \subset X$, the Newtonian space $N^{1, p}(U)$ is defined by considering $\left(U,\left.d\right|_{U},\left.\mu\right|_{U}\right)$ as a metric space in its own right. It comes naturally with the intrinsic Sobolev capacity that we denote by $C_{p}^{U}$.

The measure $\mu$ is doubling if there exists a doubling constant $C>0$ such that for all balls $B=B\left(x_{0}, r\right):=\left\{x \in X: d\left(x, x_{0}\right)<r\right\}$ in $X$,

$$
0<\mu(2 B) \leq C \mu(B)<\infty
$$

where $\delta B=B\left(x_{0}, \delta r\right)$. A metric space with a doubling measure is proper (i.e. such that closed and bounded subsets are compact) if and only if it is complete. See Heinonen [16] for more on doubling measures.

We will also need the following definition.

Definition 2.4 The space $X$ supports a $p$-Poincaré inequality if there exist constants $C>0$ and $\lambda \geq 1$ such that for all balls $B \subset X$, all integrable functions $f$ on $X$ and all ( $p$-weak) upper gradients $g$ of $f$,

$$
f_{B}\left|f-f_{B}\right| d \mu \leq C \operatorname{diam}(B)\left(f_{\lambda B} g^{p} d \mu\right)^{1 / p},
$$


where $f_{B}:=f_{B} f d \mu:=\int_{B} f d \mu / \mu(B)$.

See Björn-Björn [4] or Heinonen-Koskela-Shanmugalingam-Tyson [19] for further discussion.

\section{Quasicontinuity and Quasiopen Sets}

We are now ready to define the two notions of quasicontinuity considered in this paper. We let $C_{p}$ denote the Sobolev capacity taken with respect to the underlying space $X$ and $C_{p}^{U}$ will be the intrinsic Sobolev capacity taken with respect to $U$, i.e. with $X$ in Definition 2.3 replaced by $U$.

Definition 3.1 Let $U \subset X$ be measurable. A function $u: U \rightarrow \overline{\mathbf{R}}:=[-\infty, \infty]$ is $C_{p}^{U}$ quasicontinuous (resp. $C_{p}$-quasicontinuous) if for every $\varepsilon>0$ there is a relatively open set $G \subset U$ such that $C_{p}^{U}(G)<\varepsilon$ (resp. an open set $G \subset X$ such that $C_{p}(G)<\varepsilon$ ) and such that $\left.u\right|_{U \backslash G}$ is finite and continuous.

This distinction was tacitly suppressed in Björn-Björn-Shanmugalingam [10], BjörnBjörn [4] and Björn-Björn-Latvala [6-8]. The first two deal only with quasicontinuous functions on open sets, and in this case the two definitions are relatively easily shown to be equivalent. In this note we show that the same equivalence holds also for quasiopen $U$ (see Definition 3.2 below), which were considered in [6-8]. The proof of this is more involved, although still rather elementary. The equivalence holds without any assumptions on the metric space other than that the measure of balls should be finite. On the other hand, the assumption that $U$ be quasiopen cannot be dropped, see Example 3.6.

Definition 3.2 A set $U \subset X$ is quasiopen if for every $\varepsilon>0$ there is an open set $G \subset X$ such that $C_{p}(G)<\varepsilon$ and $G \cup U$ is open.

Quasiopen sets are measurable by Lemma 9.3 in Björn-Björn [5]. It is also quite easy to see that every $\left(C_{p}^{U}\right.$ or $C_{p}$ )-quasicontinuous function on a measurable set (and thus in particular on a quasiopen set) is measurable.

The quasiopen sets do not (in general) form a topology. This is easily seen in unweighted $\mathbf{R}^{n}$ with $p \leq n$ as all singleton sets are quasiopen, but not all sets are quasiopen. We shall prove the following characterizations of quasiopen sets.

Proposition 3.3 Let $U \subset X$ be a quasiopen set and $V \subset U$. Then the following statements are equivalent:

(a) $\quad V$ is quasiopen (in $X$ );

(b) $\quad V$ is $C_{p}$-quasiopen in $U$, i.e. for every $\varepsilon>0$ there is a relatively open set $G \subset U$ such that $C_{p}(G)<\varepsilon$ and $G \cup V$ is relatively open in $U$;

(c) $V$ is $C_{p}^{U}$-quasiopen in $U$, i.e. for every $\varepsilon>0$ there is a relatively open set $G \subset U$ such that $C_{p}^{U}(G)<\varepsilon$ and $G \cup V$ is relatively open in $U$.

Even though the quasiopen sets do not (in general) form a topology, we still have the characterization (iii) below of quasicontinuity using quasiopen sets. 
Proposition 3.4 Let $u: U \rightarrow \overline{\mathbf{R}}$ be a function on a quasiopen set $U \subset X$. Then the following statements are equivalent:

(i) $u$ is $C_{p}^{U}$-quasicontinuous;

(ii) $u$ is $C_{p}$-quasicontinuous.

Moreover, if $X$ is locally compact then these statements are equivalent to the following statement:

(iii) $u$ is finite q.e. and the sets $U_{\alpha}:=\{x \in U: u(x)>\alpha\}$ and $V_{\alpha}:=\{x \in U$ : $u(x)<\alpha\}, \alpha \in \mathbf{R}$, are quasiopen (in any of the equivalent senses $(a),(b)$ and $(c)$ of Proposition 3.3).

Remark 3.5 The local compactness assumption is only needed when showing that there is an open neighbourhood of $\{x:|u(x)|=\infty\}$ with small capacity when proving (iii) $\Rightarrow$ (ii). In particular (i) $\Leftrightarrow$ (ii) $\Leftrightarrow$ (iii) holds without this assumption for real-valued functions $u: U \rightarrow \mathbf{R}$.

Example 3.6 The equivalence between the two types of quasicontinuity does not hold for arbitrary measurable subsets: To see this let e.g. $U=(\mathbf{R} \backslash \mathbf{Q})^{2}$ as a subset of (unweighted) $\mathbf{R}^{2}$ and $u=\chi_{[0, \sqrt{2}]^{2}}$. Then any relatively open set $G \subset U$ such that $\left.u\right|_{U \backslash G}$ is continuous must contain at least one point on each line $\{(x, y): x=t\}$ for $0<t<\sqrt{2}, t \notin \mathbf{Q}$. It follows, by projection, that $C_{p}(G) \geq C_{p}(([0, \sqrt{2}] \backslash \mathbf{Q}) \times\{0\})>0$ and thus $u$ is not $C_{p^{-}}$ quasicontinuous. On the other hand, let $E=U \cap(([0, \sqrt{2}] \times\{\sqrt{2}\}) \cup(\{\sqrt{2}\} \times[0, \sqrt{2}]))$. Then $\left.u\right|_{U \backslash E}$ is continuous. Since $\mu(E)=0$, the regularity of the measure shows that, for every $\varepsilon>0$, there is a relatively open subset $G$ of $U$ such that $E \subset G$ and $\mu(G)<\varepsilon$. As there are no nonconstant curves in $U$, we have $C_{p}^{U}(G)=\mu(G)$, and thus $u$ is $C_{p}^{U}$ quasicontinuous.

In Fuglede [13, Lemma 3.3], a characterization similar to (iii) was obtained for general capacities on topological spaces. The definitions of quasiopen sets and quasicontinuity therein differ however somewhat from ours. More precisely, in [13], a set $V$ is called quasiopen if for every $\varepsilon>0$ there exists an open set $\Omega$ such that the symmetric difference $(V \backslash \Omega) \cup(\Omega \backslash V)$ has capacity less than $\varepsilon$. If the capacity is outer then this notion is easily shown to be equivalent to our definition, but in general Fuglede's definition allows for more quasiopen sets.

Similarly, in the definition of quasicontinuity in [13], it is not in general required that the removed exceptional set $G$ be open (though for outer capacities this can always be arranged) and continuity does not require finiteness. In other words, Fuglede's definition of quasicontinuity corresponds to the weak quasicontinuity considered (on open sets) in Björn-Björn [4, Section 5.2], less the requirement that continuous functions be finite. Fuglede's notion of quasicontinuity is in [13, Lemma 3.3] proved to be equivalent to the fact that the sets $U_{\alpha}$ and $V_{\alpha}, \alpha \in \mathbf{R}$, in Proposition 3.4 are quasiopen (in the sense of [13]).

Since our definitions do not exactly agree with those in [13], and moreover we consider quasicontinuity and quasiopenness with respect to two different capacities $\left(C_{p}\right.$ and $\left.C_{p}^{U}\right)$ and two possible underlying spaces ( $X$ and $U$ ), we present here for the reader's convenience full proofs of these results.

The proof of Proposition 3.4 can be easily modified to show that on quasiopen sets weak quasicontinuity is the same with respect to $C_{p}$ and $C_{p}^{U}$. 
Proof of Proposition 3.3 (a) $\Rightarrow$ (b) For every $\varepsilon>0$ there exists an open $G \subset X$ such that $C_{p}(G)<\varepsilon$ and $\Omega:=V \cup G$ is open. Then $\widetilde{G}:=G \cap U$ and $\widetilde{\Omega}:=\Omega \cap U$ are relatively open in $U$ and $C_{p}(\widetilde{G})<\varepsilon$, i.e. (b) holds.

(b) $\Rightarrow$ (c) This is straightforward, since $C_{p}^{U}$ is majorized by $C_{p}$.

(c) $\Rightarrow$ (a) We may assume that $\emptyset \neq U \neq X$. Let $\varepsilon>0$ be arbitrary. Since $U$ is quasiopen, there is an open set $G$ such that $C_{p}(G)<\varepsilon$ and such that $\Omega:=U \cup G \neq X$ is open. Hence there is $w \in N^{1, p}(X)$ such that $\chi_{G} \leq w \leq 1$ and $\|w\|_{N^{1, p}(X)}^{p}<\varepsilon$.

Using that $V$ is $C_{p}^{U}$-quasiopen, we can find, for each $j=1,2, \ldots$, a relatively open set $G_{j} \subset U$ such that $V \cup G_{j}$ is relatively open in $U$ and $C_{p}^{U}\left(G_{j}\right)<\varepsilon_{j}:=2^{-j} j^{-p} \varepsilon$. There is thus $v_{j} \in N^{1, p}(U)$ such that $\chi_{G_{j}} \leq v_{j} \leq 1$ in $U$ and $\left\|v_{j}\right\|_{N^{1, p}(U)}^{p}<\varepsilon_{j}$. Next, let $x_{0} \in \Omega$,

$$
\Omega_{j}=\left\{x \in \Omega: \operatorname{dist}(x, X \backslash \Omega)>1 / j \text { and } d\left(x, x_{0}\right)<j\right\}, \quad j=1,2, \ldots,
$$

and $\eta_{j}(x)=\left(1-j \operatorname{dist}\left(x, \Omega_{j}\right)\right)_{+}$. (If $\Omega_{j}=\emptyset$ we let $\left.\eta_{j} \equiv 0\right)$. Define the functions

$$
\varphi_{k}=\left\{\begin{array}{ll}
\min \left\{1-w, \max _{1 \leq j \leq k} v_{j} \eta_{j}\right\} & \text { in } U, \\
0, & \text { otherwise, }
\end{array} \quad \text { and } \varphi=\lim _{k \rightarrow \infty} \varphi_{k} .\right.
$$

Then $\varphi_{k}$ has bounded support and $\varphi_{k} \in N^{1, p}(U)$. Since

$$
0 \leq \varphi_{k} \leq \min \left\{1-w, \eta_{k}\right\} \in N_{0}^{1, p}(U):=\left\{f: f \in N^{1, p}(X) \text { and } f=0 \text { outside } U\right\},
$$

Lemma 2.37 in [4] implies that $\varphi_{k} \in N_{0}^{1, p}(U) \subset N^{1, p}(X)$. Next,

$$
\int_{X} \varphi_{k}^{p} d \mu \leq \sum_{j=1}^{k} \int_{X} v_{j}^{p} d \mu<\sum_{j=1}^{k} \varepsilon_{j}<\varepsilon .
$$

By Lemma 1.52 in [4], $g:=\sup _{k} g_{\varphi_{k}}$ is a $p$-weak upper gradient of $\varphi$. For a.e. $x \in U$ we either have $g=g_{\varphi_{k}}=g_{w}$ or $g=g_{\varphi_{k}}=g_{v_{j} \eta_{j}} \leq g_{v_{j}}+j v_{j}$ for some $j$ and $k$ (see [4, Theorem 2.15 and Corollary 2.21]). Hence

$$
\int_{X} g^{p} d \mu \leq \int_{X} g_{w}^{p} d \mu+\sum_{j=1}^{\infty} 2^{p-1} \int_{U}\left(g_{v_{j}}^{p}+j^{p} v_{j}^{p}\right) d \mu \leq \varepsilon+\sum_{j=1}^{\infty} 2^{p-1} j^{p} \varepsilon_{j}<\varepsilon+2^{p} \varepsilon,
$$

which together with Eq. 3.1 shows that $\|\varphi\|_{N^{1, p}(X)}^{p} \leq 2 \varepsilon+2^{p} \varepsilon$.

Next, set

$$
H=G \cup \bigcup_{j=1}^{\infty}\left(G_{j} \cap \Omega_{j}\right),
$$

which is open by the choice of $G$ as $\bigcup_{j=1}^{\infty}\left(G_{j} \cap \Omega_{j}\right)$ is relatively open in $U$. Then $w+\varphi \geq$ $\chi_{H}$ and hence

$$
C_{p}(H) \leq\|w+\varphi\|_{N^{1, p}(X)}^{p} \leq 2^{p-1}\left(\|w\|_{N^{1, p}(X)}^{p}+\|\varphi\|_{N^{1, p}(X)}^{p}\right) \leq 2^{p-1}\left(3 \varepsilon+2^{p} \varepsilon\right) .
$$

It remains to show that $V \cup H$ is open in $X$. If $x \in V$, then $x \in \Omega_{k}$ for some $k$, and as $V \cup G_{k}$ is relatively open in $U$ (and thus $G \cup V \cup G_{k}$ is open in $X$ ), we can find a ball $B_{x}$ such that

$$
x \in B_{x} \subset\left(G \cup V \cup G_{k}\right) \cap \Omega_{k} \subset V \cup G \cup\left(G_{k} \cap \Omega_{k}\right) \subset V \cup H .
$$

For $x \in H$, we can instead choose $x \in B_{x} \subset H \subset V \cup H$. 
Proof of Proposition 3.4 (ii) $\Rightarrow$ (i) This is straightforward, since $C_{p}^{U}$ is majorized by $C_{p}$.

(i) $\Rightarrow$ (ii) We may assume that $\emptyset \neq U \neq X$. Let $\varepsilon>0$ be arbitrary. For each $j=1,2, \ldots$, there is a relatively open set $G_{j} \subset U$ such that $C_{p}^{U}\left(G_{j}\right)<\varepsilon_{j}:=2^{-j} j^{-p} \varepsilon$ and such that $\left.u\right|_{U \backslash G_{j}}$ is finite and continuous in $U$.

Next construct the open sets $G, H$ and $\Omega_{k}, k=1,2, \ldots$, as in the proof of Proposition 3.3, (c) $\Rightarrow$ (a). Then $C_{p}(H) \leq 2^{p-1}\left(3 \varepsilon+2^{p} \varepsilon\right)$. If $x \in U \backslash H$, then there is $k$ such that $x \in \Omega_{k}$. Since $\left.u\right|_{U \backslash G_{k}}$ is finite and continuous at $x$, it thus follows that $\left.u\right|_{U \backslash H}$ is finite and continuous at $x$. Hence $\left.u\right|_{U \backslash H}$ is finite and continuous, and $u$ is $C_{p}$-quasicontinuous.

Now assume that $X$ is locally compact.

(ii) $\Rightarrow$ (iii) Let $\varepsilon>0$. Since $U$ is quasiopen we can find an open set $G$ such that $G \cup U$ is open and $C_{p}(G)<\varepsilon$. As $u$ is $C_{p}$-quasicontinuous, there is an open set $H \subset X$ such that $C_{p}(H)<\varepsilon$ and $\left.u\right|_{U \backslash H}$ is continuous. Thus, for every $\alpha \in \mathbf{R}, U_{\alpha} \backslash H$ is relatively open in $U \backslash H$. Hence $U_{\alpha} \cup(G \cup H)$ is open and $C_{p}(G \cup H)<2 \varepsilon$, showing that $U_{\alpha}$ is quasiopen. That $V_{\alpha}$ is quasiopen follows similarly, whereas $u$ is finite q.e. by definition.

(iii) $\Rightarrow$ (ii) Let $\varepsilon>0$ and $E=\{x \in U:|u(x)|=\infty\}$. By assumption, $C_{p}(E)=0$ and thus there is an open set $H \supset E$ such that $C_{p}(H)<\varepsilon$, by Proposition 1.4 in BjörnBjörn-Shanmugalingam [10] and Proposition 4.7 in Björn-Björn-Lehrbäck [9]. Next let $\left\{q_{j}\right\}_{j=1}^{\infty}$ be an enumeration of $\mathbf{Q}$. By assumption there are open $G_{j}$ such that $U_{q_{j}} \cup G_{j}$ and $V_{q_{j}} \cup G_{j}$ are open and $C_{p}\left(G_{j}\right)<2^{-j} \varepsilon$. Let $G=H \cup \bigcup_{j=1}^{\infty} G_{j}$, which is open and such that $C_{p}(G)<2 \varepsilon$. Moreover, $U_{q_{j}} \backslash G$ is relatively open in $U \backslash G$. For $\alpha \in \mathbf{R}$ it follows that

$$
U_{\alpha} \backslash G=\bigcup_{\mathbf{Q} \ni q>\alpha}\left(U_{q} \backslash G\right)
$$

is relatively open in $U \backslash G$. Similarly $V_{\alpha} \backslash G$ is relatively open in $U \backslash G$, and thus $\left.u\right|_{U \backslash G}$ is finite and continuous.

Theorem 1.1 shows that under certain assumptions on $X, p$-path open sets are quasiopen, and thus Propositions 3.3 and 3.4 hold for $p$-path open sets in that case. In general, $p$-path open sets need not be measurable, see Example 4.7 below. It would therefore be interesting to know if the conclusions of Propositions 3.3 and 3.4 hold for measurable $p$-path open sets $U$ (or even measurable $p$-path almost open sets $U$, see Björn-Björn [5]) without additional assumptions on $X$. In Section 5 some partial results are obtained for $p$-path open sets which are Borel. Note that in the situation described in Example 4.7 below, the conclusions of Propositions 3.3 and 3.4 do hold for measurable $p$-path open sets.

\section{4 p-Path Open and Quasiopen Sets}

Definition 4.1 A set $G \subset X$ is $p$-path open (in $X$ ) if for $p$-almost every curve $\gamma:\left[0, l_{\gamma}\right] \rightarrow$ $X$, the set $\gamma^{-1}(G)$ is (relatively) open in $\left[0, l_{\gamma}\right]$.

The arguments proving Propositions 3.3 and 3.4 can also be used to show that $C_{p}$ and $C_{p}^{U}$ have the same zero sets for quasiopen $U$. Using a different approach we can obtain this more generally for $p$-path open sets. 
Proposition 4.2 Let $U$ be a measurable p-path open set and $E \subset U$. Then $C_{p}(E)=0$ if and only if $C_{p}^{U}(E)=0$.

For open $U$ this is well-known, see e.g. Lemma 2.24 in Björn-Björn [4]. To prove this proposition we will need the following lemma.

Lemma 4.3 Let $\Gamma_{E}^{U}$ be the set of curves $\gamma:\left[0, l_{\gamma}\right] \rightarrow U$ which hit $E \subset U$, i.e. $\gamma^{-1}(E) \neq$ $\emptyset$. If $U$ is p-path open and $E \subset U$, then $\operatorname{Mod}_{p}\left(\Gamma_{E}^{U}\right)=\operatorname{Mod}_{p}\left(\Gamma_{E}^{X}\right)$.

If $U$ is not $p$-path open, then this is not true in general: Consider, e.g., $E=\{0\} \subset \mathbf{R}$ and $U=\mathbf{Q}$, in which case $\operatorname{Mod}_{p}\left(\Gamma_{E}^{U}\right)=0<\operatorname{Mod}_{p}\left(\Gamma_{E}^{\mathbf{R}}\right)$.

Proof Since $\Gamma_{E}^{U} \subset \Gamma_{E}^{X}$, we have $\operatorname{Mod}_{p}\left(\Gamma_{E}^{U}\right) \leq \operatorname{Mod}_{p}\left(\Gamma_{E}^{X}\right)$.

Conversely, as $U$ is $p$-path open, $p$-almost every curve $\gamma \in \Gamma_{E}^{X}$ is such that $\gamma^{-1}(U)$ is relatively open in $\left[0, l_{\gamma}\right]$ (and we can ignore the other curves in $\Gamma_{E}^{X}$ ). Moreover $\gamma^{-1}(U) \supset$ $\gamma^{-1}(E) \neq \emptyset$. Hence $\gamma^{-1}(U)$ is a nonempty countable union of relatively open intervals of $\left[0, l_{\gamma}\right]$, at least one of which contains a point $t \in \gamma^{-1}(E)$. We can thus find a small compact interval $[a, b] \ni t, 0 \leq a<b \leq l_{\gamma}$, such that $[a, b] \subset \gamma^{-1}(U)$. Then $\left.\gamma\right|_{[a, b]} \in \Gamma_{E}^{U}$, and Lemma 1.34 (c) in [4] implies that $\operatorname{Mod}_{p}\left(\Gamma_{E}^{X}\right) \leq \operatorname{Mod}_{p}\left(\Gamma_{E}^{U}\right)$.

Proof of Proposition 4.2 Assume that $C_{p}^{U}(E)=0$. Proposition 1.48 in [4], applied with $U$ as the underlying space, implies that $\mu(E)=0$ and that $p$-almost every curve in $U$ avoids $E$. Since $U$ is $p$-path open, it follows from Lemma 4.3 that also $p$-almost every curve in $X$ avoids $E$. Thus, by Proposition 1.48 in [4] again (this time with respect to $X$ ), $C_{p}(E)=0$.

The converse implication is trivial.

The $p$-path open sets were introduced by Shanmugalingam [29, Remark 3.5]. It was also shown there that every quasiopen set is $p$-path open. We are now going to prove Theorem 1.1 which says that the converse is true under suitable assumptions on $X$. In particular, this holds in $\mathbf{R}^{n}$.

Proof of Theorem 1.1 Let $U \subset X$ be $p$-path open. Then the family $\Gamma$ of curves $\gamma$ in $X$, for which $\gamma^{-1}(U)$ is not relatively open, has zero $p$-modulus, i.e. there exists $\rho \in L^{p}(X)$ such that $\int_{\gamma} \rho d s=\infty$ for every $\gamma \in \Gamma$.

Assume to start with that $U$ is bounded and let $B$ be a ball containing $U$. Define, for $x \in X$,

$$
u(x)=\min \left\{1, \inf _{\gamma} \int_{\gamma}\left(\rho+\chi_{B}\right) d s\right\},
$$

where $\chi_{B}$ is the characteristic function of $B$ and the infimum is taken over all rectifiable curves $\gamma:\left[0, l_{\gamma}\right] \rightarrow X$ (including constant curves) such that $\gamma(0)=x$ and $\gamma\left(l_{\gamma}\right) \in$ $X \backslash U$. Then $u=0$ in $X \backslash U$ and Lemma 3.1 in Björn-Björn-Shanmugalingam [10] (or [4, Lemma 5.25]) shows that $u$ has $\rho+\chi_{B}$ as an upper gradient. Since the measure $\mu$ is doubling and $X$ is complete and supports a $p$-Poincaré inequality, we can conclude from Theorem 1.11 in Järvenpää-Järvenpää-Rogovin-Rogovin-Shanmugalingam [20] that $u$ is measurable. As $U$ is assumed to be bounded and $\rho \in L^{p}(X)$, it follows that $u \in N^{1, p}(X)$.

We claim that $u>0$ in $U$, i.e. that $U=\{x \in X: u(x)>0\}$ is a superlevel set of a Newtonian function. The assumptions on $X$ guarantee that $u$ is quasicontinuous (by [10, Theorem 1.1] or [4, Theorem 5.29]), which then directly implies that $U$ is quasiopen, see Proposition 3.4. 
To prove the claim, let $x \in U$ and assume for a contradiction that $u(x)=0$. Then there exist curves $\gamma_{j}$ connecting $x$ to $X \backslash U$ such that

$$
\int_{\gamma_{j}}\left(\rho+\chi_{B}\right) d s \leq 2^{-j}, \quad j=1,2, \ldots
$$

In particular, $l_{\gamma_{j}} \leq 2^{-j}$ for all $j=1,2, \ldots$.

We define a curve $\tilde{\gamma}$ as a recursive concatenation of all $\gamma_{j}$ and their reversed reparameterizations as follows. Let $L_{0}=0$,

$$
L_{j}=2 \sum_{i=1}^{j} l_{\gamma_{i}} \leq 2 \sum_{i=1}^{\infty} l_{\gamma_{i}}=: L \leq 2 \sum_{j=1}^{\infty} 2^{-j}=2
$$

and

$$
\tilde{\gamma}(t)= \begin{cases}\gamma_{j}\left(t-L_{j-1}\right), & \text { if } L_{j-1} \leq t \leq L_{j-1}+l_{\gamma_{j}}, j=1,2, \ldots, \\ \gamma_{j}\left(L_{j}-t\right), & \text { if } L_{j-1}+l_{\gamma_{j}} \leq t \leq L_{j}, j=1,2, \ldots\end{cases}
$$

Then $\tilde{\gamma}:[0, L] \rightarrow X, \tilde{\gamma}(L)=x$ and $\tilde{\gamma}\left(L_{j}+l_{\gamma_{j+1}}\right) \in X \backslash U, j=1,2, \ldots$ Since $x \in U$ and $L_{j}+l_{\gamma_{j+1}} \rightarrow L$, as $j \rightarrow \infty$, this shows that $\tilde{\gamma}^{-1}(U)$ is not relatively open in $[0, L]$ and hence $\tilde{\gamma} \in \Gamma$. But Eq. 4.2 implies that

$$
\int_{\tilde{\gamma}} \rho d s \leq 2 \sum_{j=1}^{\infty} \int_{\gamma_{j}}\left(\rho+\chi_{B}\right) d s \leq 2 \sum_{j=1}^{\infty} 2^{-j}=2,
$$

contradicting the choice of $\rho$. We can therefore conclude that $u(x)>0$ for all $x \in U$, which finishes the proof for bounded $U$.

If $U$ is unbounded, then the above argument shows that $U \cap B_{j}$ is quasiopen, $j=$ $1,2, \ldots$, where $\left\{B_{j}\right\}_{j=1}^{\infty}$ is a countable cover of $X$ by (open) balls. In particular, given $\varepsilon>0$, there exists for every $j=1,2, \ldots$ an open set $G_{j}$ such that $C_{p}\left(G_{j}\right)<2^{-j} \varepsilon$ and $(U \cap$ $\left.B_{j}\right) \cup G_{j}$ is open. Setting $G=\bigcup_{j=1}^{\infty} G_{j}$, we see that $C_{p}(G)<\varepsilon$ and $U \cup G=\bigcup_{j=1}^{\infty}((U \cap$ $\left.B_{j}\right) \cup G_{j}$ ) is open, which concludes the proof.

We now turn to Theorem 1.2 and restate it in a more general form.

Proposition 4.4 Assume that $X$ is locally compact and that every measurable p-path open set in $X$ is quasiopen. Let $u: U \rightarrow \overline{\mathbf{R}}$ be a function on a quasiopen set $U$. Then the following are equivalent:

(a) $u$ is quasicontinuous (with respect to $C_{p}$ or $C_{p}^{U}$ );

(b) $u$ is measurable and finite q.e., and $u \circ \gamma$ is continuous (on the set where it is defined) for $p$-a.e. curve $\gamma:\left[0, l_{\gamma}\right] \rightarrow X$;

(c) $u$ is measurable and finite q.e., and $u \circ \gamma$ is continuous for $p$-a.e. curve $\gamma:\left[0, l_{\gamma}\right] \rightarrow$ $U$.

The assumptions of Proposition 4.4 are guaranteed by Theorem 1.1, but there are also other situations when they hold, see e.g. Example 4.7 below and Example 5.6 in [4].

As seen from the proof below and Remark 3.5, no assumptions on $X$ are needed for the implications (a) $\Rightarrow$ (b) $\Leftrightarrow$ (c) in Proposition 4.4. Also, if we know that every $p$-path open set is quasiopen (and thus measurable), then the measurability assumption for $u$ can be dropped, cf. the proof of Theorem 1.1 where measurability of $u$ follows from Theorem 1.11 in Järvenpää-Järvenpää-Rogovin-Rogovin-Shanmugalingam [20]. 
For real-valued $u$, the assumption of local compactness here and in Corollary 4.5 below can be omitted, see Remark 3.5.

Proof (a) $\Rightarrow$ (b) Assume that $u: U \rightarrow \overline{\mathbf{R}}$ is quasicontinuous. Proposition 3.4 shows that it is finite q.e. and the sets $U_{\alpha}:=\{x \in U: u(x)>\alpha\}$ and $V_{\alpha}:=\{x \in U: u(x)<\alpha\}, \alpha \in \mathbf{R}$, are quasiopen. Remark 3.5 in Shanmugalingam [29] implies that $U, U_{\alpha}$ and $V_{\alpha}, \alpha \in \mathbf{R}$, are $p$-path open. Hence, for $p$-a.e. curve $\gamma:\left[0, l_{\gamma}\right] \rightarrow X$, the set $I_{\gamma}=\gamma^{-1}(U)$ is a relatively open subset of $\left[0, l_{\gamma}\right]$, and so are the level sets

$$
\gamma^{-1}\left(U_{\alpha}\right)=\left\{t \in I_{\gamma}:(u \circ \gamma)(t)>\alpha\right\} \quad \text { and } \quad \gamma^{-1}\left(V_{\alpha}\right)=\left\{t \in I_{\gamma}:(u \circ \gamma)(t)<\alpha\right\} \text {, }
$$

for all $\alpha \in \mathbf{Q}$. This implies that $u \circ \gamma: I_{\gamma} \rightarrow \overline{\mathbf{R}}$ is continuous for $p$-a.e. curve $\gamma$.

(b) $\Rightarrow$ (c) This is trivial.

(c) $\Rightarrow$ (b) Let $\Gamma$ be the family of exceptional curves $\gamma:\left[0, l_{\gamma}\right] \rightarrow U$ for which $u \circ \gamma$ is not continuous. As $U$ is quasiopen, and thus $p$-path open by Remark 3.5 in [29], $\gamma^{-1}(U)$ is open for $p$-a.e. curve $\gamma:\left[0, l_{\gamma}\right] \rightarrow X$. By Lemma 1.34 in [4], $p$-a.e. such curve $\gamma$ does not have a subcurve in $\Gamma$. For such a $\gamma$, the composition $u \circ \gamma$ is continuous on the relatively open subset of $\left[0, l_{\gamma}\right]$ where it is defined.

(b) $\Rightarrow$ (a) Since $U$ is quasiopen, and thus $p$-path open by Remark 3.5 in [29], for $p$-a.e. curve $\gamma:\left[0, l_{\gamma}\right] \rightarrow X$, the set $I_{\gamma}=\gamma^{-1}(U)$ is a relatively open subset of $\left[0, l_{\gamma}\right]$ and $u \circ \gamma$ is continuous on $I_{\gamma}$. For such $\gamma$, and all $\alpha \in \mathbf{R}$, the level sets in Eq. 4.3 are relatively open in $I_{\gamma}$, and thus in $\left[0, l_{\gamma}\right]$. It follows that the level sets $U_{\alpha}:=\{x \in U: u(x)>\alpha\}$ and $V_{\alpha}:=\{x \in U: u(x)<\alpha\}, \alpha \in \mathbf{R}$, are $p$-path open and measurable (as $u$ is measurable), and thus quasiopen by the assumption. Proposition 3.4 now concludes the proof.

Proof of Theorem 1.2 Theorem 1.1 guarantees that the assumptions of Proposition 4.4 are satisfied, from which the result follows.

As a consequence of the characterization in Proposition 4.4 we obtain the following generalization of Theorem 1.3 and partial converse of Theorem 1.4.

Corollary 4.5 Assume that $X$ is locally compact and that every measurable p-path open set in $X$ is quasiopen. Let $U \subset X$ be quasiopen. Then all $u \in N_{\mathrm{loc}}^{1, p}(U)$ are quasicontinuous.

Note that in particular we may let $U=X$.

Proof Since every Newtonian function is measurable, finite q.e. (by [4, Proposition 1.30]) and absolutely continuous on $p$-a.e. curve, by Shanmugalingam [28, Proposition 3.1] (or [4, Theorem 1.56]), this follows directly from Proposition 4.4.

\section{Proof of Theorem 1.3 This follows directly from Theorem 1.1 and Corollary 4.5.}

Remark 4.6 Theorem 1.3 was recently obtained by Björn-Björn-Latvala [7]. The proof given here is very different from that in [7] and appears as an immediate corollary of other results. In particular, it does not use the fine topology and the quasi-Lindelöf principle, whose proof in [7] relies on the vector-valued, so-called Cheeger, differentiable structure.

The assumptions of our Corollary 4.5 are weaker than those of the version of Theorem 1.3 in [7]. On the other hand, in [7], quasicontinuity is deduced for a larger local space than $N_{\text {loc }}^{1, p}(U)$. See [7] for more details and the precise definitions of the local spaces considered therein. 
The following example shows that $p$-path open sets need not be quasiopen in general.

Example 4.7 Assume that there are no nonconstant rectifiable curves in $X$. For example, consider $\mathbf{R}$ with the snowflaked metric $d(x, y)=|x-y|^{\alpha}, 0<\alpha<1$, and the Lebesgue measure.

Then every set is $p$-path open, while Lemma 9.3 in Björn-Björn [5] shows that quasiopen sets must be measurable. Any nonmeasurable set $U \subset X$ is thus $p$-path open but cannot be quasiopen. Indeed, the function $u$ constructed in the proof of Theorem 1.1 is $\chi_{U}$, which has zero as an upper gradient, but it is not measurable and thus not in $N^{1, p}(X)$.

Note that in this case the zero function is an upper gradient of every function and thus $N^{1, p}(X)=L^{p}(X)$ and $C_{p}$ is the extension of the measure $\mu$ to all subsets of $X$ as an outer measure. It thus follows that the quasiopen sets are just the measurable sets, and hence every measurable p-path open set is quasiopen. Thus Corollary 4.5 applies in this case, but this already follows (in this particular case) from Luzin's theorem.

The same argument applies also if the family of nonconstant rectifiable curves has zero $p$-modulus. (In this case "upper gradient" should be replaced by " $p$-weak upper gradient" above). In fact, this assumption is equivalent to the equality $N^{1, p}(X)=L^{p}(X)$ as sets of functions, see L. Malý [26, Lemma 2.5].

\section{Outside the Realm of a Poincaré Inequality}

The doubling condition and the Poincaré inequality are standard assumptions in analysis on metric spaces. In particular, they guarantee that Lipschitz functions are dense in $N^{1, p}(X)$, which in turn (together with completeness) implies the quasicontinuity of Newtonian functions used in the proof of Theorem 1.1. On the other hand, there are plenty of spaces where the Poincaré inequality fails or the doubling condition is violated, but where Newtonian functions are quasicontinuous. Therefore, in this section we relax the assumptions of Theorem 1.1 and obtain Theorem 1.4.

We postpone the proof of Theorem 1.4 to the end of this section. Meanwhile, we formulate some consequences of it. First, we have the following characterization of quasicontinuity among Borel functions, analogous to Proposition 4.4. Note that the assumption of quasiopenness of $p$-path open sets can be deduced using Theorem 1.4, or its generalization Theorem 5.7 below, under the assumptions therein.

Proposition 5.1 Assume that $X$ is locally compact and that every Borel p-path open set in $X$ is quasiopen. Let $u: U \rightarrow \overline{\mathbf{R}}$ be a Borel function on a quasiopen set $U$. Then the following are equivalent:

(a) $u$ is quasicontinuous (with respect to $C_{p}$ or $C_{p}^{U}$ );

(b) $u$ is finite q.e. and $u \circ \gamma$ is continuous (on the set where it is defined) for p-a.e. curve $\gamma:\left[0, l_{\gamma}\right] \rightarrow X$

(c) $u$ is finite q.e. and $u \circ \gamma$ is continuous for p-a.e. curve $\gamma:\left[0, l_{\gamma}\right] \rightarrow U$.

Proof As mentioned before the proof of Proposition 4.4, the proof of (a) $\Rightarrow$ (b) $\Leftrightarrow$ (c) therein holds without any assumptions on $X$ and therefore applies also here. 
(b) $\Rightarrow$ (a) Replace the word "measurable" by "Borel" twice in the proof of this implication in the proof of Proposition 4.4.

Corollary 5.2 Assume that $X$ is complete and locally compact. Consider the following statements:

(a) every bounded $u \in N^{1, p}(X)$ is quasicontinuous;

(b) every $u \in N_{\mathrm{loc}}^{1, p}(X)$ is quasicontinuous;

(c) every Borel p-path open set in $X$ is quasiopen;

(d) if $U$ is quasiopen, then every Borel function $u \in N_{\text {loc }}^{1, p}(U)$ is quasicontinuous.

Then $(a) \Leftrightarrow(b) \Rightarrow(c) \Rightarrow(d)$.

Moreover, if every bounded function $u \in N^{1, p}(X)$ has a Borel representative $\tilde{u} \in$ $N^{1, p}(X)$, then all the statements are equivalent.

Recall that $\tilde{u}$ is termed a representative of $u$ if both functions belong to the same equivalence class in $N^{1, p}(X) / \sim$ (or, equivalently, if $\tilde{u}=u$ q.e., see Section 2 ).

Proof (a) $\Rightarrow$ (b) Let $u \in N_{\text {loc }}^{1, p}(X)$. Fix $x_{0} \in X$ and let $\eta_{j} \in \operatorname{Lip}(X)$ be such that $\eta_{j}=1$ on $B\left(x_{0}, j\right)$ and $\eta_{j}=0$ outside $B\left(x_{0}, 2 j\right)$. Then $u_{j}:=u \eta_{j} \in N^{1, p}(X)$ and also $\arctan u_{j} \in$ $N^{1, p}(X)$. By assumption $\arctan u_{j}$ is quasicontinuous. As $u_{j}$ is finite q.e., Proposition 1.4 in [10] and Proposition 4.7 in [9] show that also $u_{j}$ is quasicontinuous in $X$. Hence $u$ is quasicontinuous in $B\left(x_{0}, j\right)$ for each $j$, and it follows from Lemma 5.18 in [4] that $u$ is quasicontinuous in $X$.

(b) $\Rightarrow$ (a) This is trivial.

(a) $\Rightarrow$ (c) This is Theorem 1.4.

(c) $\Rightarrow$ (d) Let $u \in N_{\text {loc }}^{1, p}(U)$ be a Borel function. Then it is finite q.e. and absolutely continuous on $p$-a.e. curve (see Shanmugalingam [28, Proposition 3.1] or Proposition 1.30 and Theorem 1.56 in [4]). The quasicontinuity of $u$ then follows from Proposition 5.1.

Finally, assume that every bounded function $u \in N^{1, p}(X)$ has a Borel representative $\tilde{u} \in$ $N^{1, p}(X)$ and that (d) holds with $U=X$. Let $u \in N^{1, p}(X)$ be bounded and $\tilde{u} \in N^{1, p}(X)$ be a Borel representative of $u$. By assumption $\tilde{u}$ is quasicontinuous. As $X$ is locally compact, Proposition 1.4 in Björn-Björn-Shanmugalingam [10] (or [4, Proposition 5.27]) and Proposition 4.7 in Björn-Björn-Lehrbäck [9] show that also $u$ is quasicontinuous. Hence (a) holds.

The following open problems are natural in view of Corollary 5.2 and the comment after it.

Open problem 5.3 Does every (bounded) $u \in N^{1, p}(X)$ have a Borel representative?

Note that if every bounded $u \in N^{1, p}(X)$ has a Borel representative, then also every unbounded $v \in N^{1, p}(X)$ has a Borel representative. Indeed, if $u=\arctan v$ has a Borel representative $\tilde{u}$ (which we may require to have values in $(-\pi / 2, \pi / 2)$ ), then $\tan \tilde{u}$ becomes a Borel representative of $v$.

Since any quasiopen set can be written as a union of a Borel set and a set of capacity zero (cf. [8, Lemma 9.5]), Proposition 3.4 shows that the answer to Open problem 5.3 is positive for a particular space $X$ if all Newtonian functions on $X$ are quasicontinuous. 
Open problem 5.4 Can "Borel" in Theorem 1.4 be replaced by "measurable"? Example 4.7 shows that it cannot be omitted altogether.

It would follow that a version of Corollary 5.2 with "Borel" replaced by "measurable" would also be possible, and since all Newtonian functions are measurable, the equivalence of (a)-(d) therein would follow in that case.

We now proceed to the proof of Theorem 1.4. For this, we will need Proposition 1.5 about measurability of the function $u$ in Eq. 4.1, which does not rely on any Poincaré inequality.

Recall that a subset of a complete separable metric space is analytic (or Suslin) if it is a continuous image of a complete separable metric space, see e.g. Kechris [21, Definitions 3.1 and 14.1]. By Theorem 14.11 (Suslin's theorem) therein, every Borel subset of a complete separable space is analytic. (In fact, it shows that Borel sets are exactly those analytic sets which are also coanalytic, i.e. whose complements are analytic).

Proposition 14.4 in [21] tells us that countable unions and countable intersections of analytic sets are analytic. Moreover, if $f: Y \rightarrow Z$ is a Borel mapping between two complete separable metric spaces, then images and preimages under $f$ of analytic sets are analytic (also by Proposition 14.4 in [21]). By Theorem 21.10 in [21] (Luzin's theorem), every analytic subset of a complete separable metric space $Y$ is $\nu$-measurable for every $\sigma$-finite Borel measure $v$ on $Y$.

When proving Theorem 1.4 we will need Proposition 1.5 with a lower semicontinuous $\rho$ only, in which case the proof can be considerably simplified (in particular the use of Lemma 5.5 can be avoided). As we find it interesting that Proposition 1.5 is true also for Borel functions we give a proof of the more general result, for which we need the following result from Järvenpä-Järvenpä-Rogovin-Rogovin-Shanmugalingam [20, Lemma 2.4].

Lemma 5.5 Let $Z$ be a metric space and let $\mathcal{Y}$ be a class of functions $\rho: Z \rightarrow[0, \infty]$ such that the following properties hold:

(a) $\mathcal{Y}$ contains all continuous $\rho: Z \rightarrow[0, \infty]$;

(b) if $\rho_{j} \in \mathcal{Y}$ and $\rho_{j} \nearrow \rho$ then $\rho \in \mathcal{Y}$;

(c) if $\rho, \sigma \in \mathcal{Y}$ and $r, s \in \mathbf{R}^{+}$then $r \rho+s \sigma \in \mathcal{Y}$;

(d) if $\rho \in \mathcal{Y}$ and $0 \leq \rho \leq 1$ then $1-\rho \in \mathcal{Y}$.

Then $\mathcal{Y}$ contains all Borel functions $\rho: Z \rightarrow[0, \infty]$.

Proof of Proposition 1.5 For $\alpha>0$, let $\mathcal{L}_{\alpha}$ consist of all continuous curves $\gamma:[0,1] \rightarrow X$ with $\operatorname{Lip} \gamma \leq \alpha$. (In this proof we want all curves parameterized on the same interval, and do not assume that they are parameterized by arc length). Then $\mathcal{L}_{\alpha}$ is a metric space with respect to the supremum norm. Since $X$ is complete, it follows from Ascoli's theorem that $\mathcal{L}_{\alpha}$ is complete. (See e.g. Royden [27, p. 169] for a version of Ascoli's theorem valid for metric space valued equicontinuous functions).

As $X$ is separable, it is easily verified that $\mathcal{L}_{\alpha}$ is separable. Indeed, let $\mathcal{U}$ be a countable base of the topology on $X$ and let $\mathcal{P}$ be the family of all finite sequences $Q=$ $\left(\left(I_{1}, U_{1}\right), \ldots,\left(I_{m}, U_{m}\right)\right)$, where $I_{i} \subset[0,1]$ are closed intervals with rational endpoints and $U_{i}$ are selected from $\mathcal{U}$. Then $\mathcal{P}$ is countable. For each $Q=\left(\left(I_{1}, U_{1}\right), \ldots,\left(I_{m}, U_{m}\right)\right) \in \mathcal{P}$, let $\mathcal{L}_{Q}$ be the family of all $\gamma \in \mathcal{L}_{\alpha}$ such that $\gamma\left(I_{i}\right) \subset U_{i}, i=1, \ldots, m$. Choosing one curve from every nonempty $\mathcal{L}_{Q}$ provides us with a countable dense system in $\mathcal{L}_{\alpha}$. 
Let $\mathcal{Y}_{\alpha}$ be the collection of all $\rho: X \rightarrow[0, \infty]$ for which the functional $\Phi_{\rho}: \mathcal{L}_{\alpha} \rightarrow$ $[0, \infty]$ defined by

$$
\Phi_{\rho}: \gamma \mapsto \int_{\gamma} \rho d s
$$

is Borel. Lemma 2.2 in Järvenpä-Järvenpä-Rogovin-Rogovin-Shanmugalingam [20] shows that if $\rho$ is continuous then $\rho \in \mathcal{Y}_{\alpha}$. If $\rho_{j} \in \mathcal{Y}$ and $\rho_{j} \nearrow \rho$ then the monotone convergence theorem implies that for all $\gamma \in \mathcal{L}_{\alpha}$,

$$
\Phi_{\rho_{j}}(\gamma)=\int_{\gamma} \rho_{j} d s \nearrow \int_{\gamma} \rho d s=\Phi_{\rho}(\gamma)
$$

i.e. that $\Phi_{\rho}$ is a limit of Borel functions on $\mathcal{L}_{\alpha}$, and hence Borel. Thus, $\rho \in \mathcal{Y}_{\alpha}$. In particular, (a) and (b) in Lemma 5.5 are satisfied by $\mathcal{Y}_{\alpha}$. The properties (c) and (d) therein follow from the linearity of the integral.

We can thus conclude from Lemma 5.5 that for every $\alpha>0$ and every Borel $\rho: X \rightarrow$ $[0, \infty]$, the functional $\Phi_{\rho}: \mathcal{L}_{\alpha} \rightarrow[0, \infty]$ is Borel. Let $\rho: X \rightarrow[0, \infty]$ be a fixed Borel function and $\alpha>0$ be arbitrary. We shall prove that the set $G=\left\{x \in X: u_{\rho}(x)<\alpha\right\}$ is measurable.

First, assume that $\rho \geq \delta$ for some $\delta>0$. Note that if $x \in G$, then for every $\gamma \in$ $\Gamma_{x}$ with $\int_{\gamma} \rho d s<\alpha$, we have $l_{\gamma}<\alpha / \delta$ and hence the reparameterized curve $\tilde{\gamma}(t):=$ $\gamma\left(l_{\gamma} t\right)$ belongs to $\mathcal{L}_{\alpha / \delta}$. It follows that for $x \in G$, the infimum in the definition of $u_{\rho}$ can equivalently be taken over all $\gamma \in \Gamma_{x} \cap \mathcal{L}_{\alpha / \delta}$. We define

$$
\Gamma_{1}=\left\{\gamma \in \mathcal{L}_{\alpha / \delta}: \int_{\gamma} \rho d s<\alpha\right\} \quad \text { and } \quad \Gamma_{2}=\left\{\gamma \in \mathcal{L}_{\alpha / \delta}: \gamma(1) \notin U\right\} .
$$

Also let $f_{j}: \mathcal{L}_{\alpha / \delta} \rightarrow X$ be the evaluation maps given by $f_{j}(\gamma)=\gamma(j), j=0,1$, which are clearly 1-Lipschitz, and thus Borel.

By the above, the functional $\Phi_{\rho}: \mathcal{L}_{\alpha / \delta} \rightarrow[0, \infty]$ is Borel, and thus $\Gamma_{1}=\Phi_{\rho}^{-1}([0, \alpha))$ is a Borel subset of $\mathcal{L}_{\alpha / \delta}$. The set $\Gamma_{2}$ is the preimage of the analytic set $X \backslash U$ under the Borel mapping $f_{1}$, and thus $\Gamma_{2}$ is analytic by Proposition 14.14 in Kechris [21]. It thus follows from Proposition 14.4 in [21] that $\Gamma_{1} \cap \Gamma_{2}$ is analytic. Since $G=f_{0}\left(\Gamma_{1} \cap \Gamma_{2}\right)$, we conclude that $G$ is analytic, and thus measurable, by Luzin's theorem [21, Theorem 21.10].

Now, let $\rho: X \rightarrow[0, \infty]$ be arbitrary and set $\rho_{j}=\rho+1 / j, j=1,2, \ldots$, . By the above, each $u_{\rho_{j}}$ is measurable. We shall show that $u_{\rho}=\lim _{j \rightarrow \infty} u_{\rho_{j}}$, which implies the measurability of $u_{\rho}$.

Given $x \in X$ and $\varepsilon>0$, there exists $\gamma \in \Gamma_{x}$ such that $\int_{\gamma} \rho d s<u_{\rho}(x)+\varepsilon$. Since $\gamma$ is rectifiable, we have

$$
u_{\rho}(x) \leq u_{\rho_{j}}(x) \leq \int_{\gamma} \rho_{j} d s \leq \int_{\gamma}(\rho+1 / j) d s<u_{\rho}(x)+\varepsilon+\frac{l_{\gamma}}{j} .
$$

Letting $j \rightarrow \infty$ and then $\varepsilon \rightarrow 0$ shows that $u_{\rho}(x)=\lim _{j \rightarrow \infty} u_{\rho_{j}}(x)$ for all $x \in X$, and we conclude that $u_{\rho}$ is measurable.

We are now ready to prove Theorem 1.4. It will be obtained in a more general form, using coanalytic sets. For separable $X$ we have the following result.

Theorem 5.6 Assume that $X$ is complete and separable, and that every bounded $u \in$ $N^{1, p}(X)$ is quasicontinuous. Then every coanalytic p-path open set $U \subset X$ is quasiopen. 
For nonseparable spaces we use the fact that $\operatorname{supp} \mu$ is always separable, by Proposition 1.6 in [4]. This way we can avoid nonseparable analytic (Suslin) sets and reduce our considerations to separable spaces where Suslin and analytic sets are the same, cf. Hansell [15] and Kechris [21]. The following result is primarily designed for nonseparable spaces. However, it improves the criterion also for separable spaces, since we do not impose any assumptions on $U \backslash \operatorname{supp} \mu$.

Theorem 5.7 Assume that supp $\mu$ is complete, and that every bounded $u \in N^{1, p}(X)$ is quasicontinuous. Then every p-path open set $U \subset X$, such that $\operatorname{supp} \mu \backslash U$ is analytic (in supp $\mu$ ), is quasiopen.

Theorem 5.6 is a special case of Theorem 5.7, but we will prove Theorem 5.6 first and then use it to prove Theorem 5.7.

Remark 5.8 By Theorem 1.1 in Björn-Björn-Shanmugalingam [10] (or [4, Theorem 5.29]) and the comments after Proposition 4.7 in Björn-Björn-Lehrbäck [9], the assumptions in Theorem 5.7 hold in particular if $X$ (or more generally supp $\mu$ ) is complete and locally compact, and continuous functions are dense in $N^{1, p}(X)$ or equivalently in $N^{1, p}(\operatorname{supp} \mu)$. The last equivalence follows from Lemma 5.19 in [4], which also implies that every (bounded) function in $N^{1, p}(X)$ is quasicontinuous if and only if every (bounded) function in $N^{1, p}(\operatorname{supp} \mu)$ is quasicontinuous.

At this point we would also like to mention that it follows from the recent results in Ambrosio-Colombo-Di Marino [2] and Ambrosio-Gigli-Savare [3] that, if supp $\mu$ is a complete doubling metric space and $1<p<\infty$, then Lipschitz functions are dense in $N^{1, p}(X)$. Thus, the assumptions on $X$ in Theorem 5.7 hold if $X$ (or $\operatorname{supp} \mu$ ) is a complete doubling metric space and $1<p<\infty$. It is easily verified that such a space is automatically locally compact.

Proof of Theorem 5.6 We follow the proof of Theorem 1.1. Let $\rho \in L^{p}(X)$ be as therein. The Vitali-Carathéodory theorem (Proposition 7.14 in Folland [12]) provides us with a lower semicontinuous pointwise majorant of $\rho$ which also belongs to $L^{p}(X)$. We can therefore without loss of generality assume that $\rho$ is lower semicontinuous.

The measurability of the function $u$ in Eq. 4.1 is now guaranteed by Proposition 1.5 and the assumption that $U$ is coanalytic, rather than by the $p$-Poincaré inequality and Theorem 1.11 in [20]. Also, since quasicontinuity of bounded Newtonian functions is assumed, it need not be concluded from the $p$-Poincaré inequality. The rest of the proof goes through verbatim.

Proof of Theorem 5.7 Let $Y=\operatorname{supp} \mu$. It follows from Proposition 1.6 in [4] that $Y$ is separable. Let $U \subset X$ be a $p$-path open set such that $Y \backslash U$ is analytic. It follows from Proposition 1.53 in [4] that $p$-almost no curve intersects $X \backslash Y$, and thus $U \cap Y$ is also $p$-path open.

Hence, as all bounded $u \in N^{1, p}(Y)$ are quasicontinuous by Lemma 5.19 in [4], Theorem 5.6 implies that $U \cap Y$ is quasiopen in $Y$, i.e. for every $\varepsilon>0$ there is a relatively open set $G \subset Y$ such that $U \cap Y \subset G$ and $C_{p}^{Y}(G \backslash U)<\varepsilon$. Thus there exists $v \in N^{1, p}(Y)$ such that $v \geq \chi_{G \backslash U}$ and $\|v\|_{N^{1, p}(Y)}^{p}<\varepsilon$. Since $p$-almost no curve intersects $X \backslash Y$ and $\mu(X \backslash Y)=0$, extending $v$ by 1 to $X \backslash Y$ shows that $C_{p}(G \backslash U) \leq\|v\|_{N^{1, p}(X)}^{p}=\|v\|_{N^{1, p}(Y)}^{p}<\varepsilon$. 
Let $G^{\prime}=G \cup(X \backslash Y)$, which is open and contains $U$. By Proposition 1.53 in [4] we see that $C_{p}(X \backslash Y)=0$ and hence

$$
C_{p}\left(G^{\prime} \backslash U\right) \leq C_{p}(G \backslash U)+C_{p}(X \backslash Y)=C_{p}(G \backslash U)<\varepsilon,
$$

showing that $U$ is quasiopen in $X$.

\section{Proof of Theorem 1.4 This is a special case of Theorem 5.7.}

Acknowledgments The first two authors were supported by the Swedish Research Council. Part of this research was done while J. B. visited the Charles University in Prague in 2014; she thanks the Department of Mathematical Analysis for support and hospitality.

Open Access This article is distributed under the terms of the Creative Commons Attribution 4.0 International License (http://creativecommons.org/licenses/by/4.0/), which permits unrestricted use, distribution, and reproduction in any medium, provided you give appropriate credit to the original author(s) and the source, provide a link to the Creative Commons license, and indicate if changes were made.

\section{References}

1. Adams, D.R., Lewis, J.L.: Fine and quasiconnectedness in nonlinear potential theory. Ann. Inst. Fourier (Grenoble) 35(1), 57-73 (1985)

2. Ambrosio, L., Colombo, M., Di Marino, S.: Sobolev spaces in metric measure spaces: reflexivity and lower semicontinuity of slope. In: Variational Methods for Evolving Objects (Sapporo, 2012), Adv. Stud. Pure Math, vol. 67, pp. 1-58, Math. Soc. Japan, Tokyo (2015)

3. Ambrosio, L., Gigli, N., Savaré, G.: Density of Lipschitz functions and equivalence of weak gradients in metric measure spaces. Rev. Mat. Iberoam 29, 969-986 (2013)

4. Björn, A., Björn, J.: Nonlinear Potential Theory on Metric Spaces. EMS Tracts in Mathematics, vol. 17, European Math. Soc., Zürich (2011)

5. Björn, A., Björn, J.: Obstacle and Dirichlet problems on arbitrary nonopen sets, and fine topology. Rev. Mat. Iberoam 31, 161-214 (2015)

6. Björn, A., Björn, J., Latvala, V.: The weak Cartan property for the p-fine topology on metric spaces. Indiana Univ. Math. J. 64, 915-941 (2015)

7. Björn, A., Björn, J., Latvala, V.: Sobolev spaces, fine gradients and quasicontinuity on quasiopen sets in $\mathbf{R}^{n}$ and metric spaces. Ann. Acad. Sci. Fenn. Math. 41, 551-560 (2016)

8. Björn, A., Björn, J., Latvala, V.: The Cartan, Choquet and Kellogg properties of the fine topology on metric spaces, to appear in J. Anal. Math.

9. Björn, A., Björn, J., Lehrbäck, J.: Sharp capacity estimates for annuli in weighted $\mathbf{R}^{n}$ and metric spaces, Preprint 17 (Fall 2013), Institut Mittag- Leffler, Djursholm

10. Björn, A., Björn, J., Shanmugalingam, N.: Quasicontinuity of Newton-Sobolev functions and density of Lipschitz functions on metric spaces. Houston J. Math. 34, 1197-1211 (2008)

11. Cheeger, J.: Differentiability of Lipschitz functions on metric measure spaces. Geom. Funct. Anal. 9, 428-517 (1999)

12. Folland, G.B.: Real Analysis, 2nd edn. Wiley, New York (1999)

13. Fuglede, B.: The quasi topology associated with a countably subadditive set function. Ann. Inst. Fourier (Grenoble) 21(1), 123-169 (1971)

14. Hajłasz, P.: Sobolev spaces on metric-measure spaces. In: Heat Kernels and Analysis on Manifolds, Graphs and Metric Spaces (Paris, 2002), Contemp. Math., vol. 338, pp. 173-218. Amer. Math. Soc., Providence (2003)

15. Hansell, R.W.: Descriptive topology. In: Recent Progress in General Topology (Prague, 1991), pp. 275315. North-Holland, Amsterdam (1992)

16. Heinonen, J.: Lectures on Analysis on Metric Spaces. Springer, New York (2001)

17. Heinonen, J., Kilpeläinen, T., Martio, O.: Nonlinear Potential Theory of Degenerate Elliptic Equations, 2nd edn. Dover, Mineola (2006)

18. Heinonen, J., Koskela, P.: Quasiconformal maps in metric spaces with controlled geometry. Acta Math. 181, 1-61 (1998) 
19. Heinonen, J., Koskela, P., Shanmugalingam, N., Tyson, J.T.: Sobolev Spaces on Metric Measure Spaces. New Mathematical Monographs, vol. 27. Cambridge Univ. Press, Cambridge (2015)

20. Järvenpää, E., Järvenpää, M., Rogovin, K., Rogovin, S., Shanmugalingam, N.: Measurability of equivalence classes and MECp-property in metric spaces. Rev. Mat. Iberoam 23, 811-830 (2007)

21. Kechris, A.S.: Classical Descriptive Set Theory. Graduate Texts in Mathematics, vol. 156. Springer, New York (1995)

22. Kilpeläinen, T., Malý, J.: Supersolutions to degenerate elliptic equations on quasi open sets. Comm. Partial Diff. Equat. 17, 371-405 (1992)

23. Koskela, P., MacManus, P.: Quasiconformal mappings and Sobolev spaces. Studia Math. 131, 1-17 (1998)

24. Latvala, V.: Finely Superharmonic Functions of Degenerate Elliptic Equations. Ann. Acad. Sci. Fenn. Ser. A I Math. Dissertationes 96 (1994)

25. Malý, J., Ziemer, W.P.: Fine Regularity of Solutions of Elliptic Partial Differential Equations. Amer. Math. Soc., Providence (1997)

26. Malý, L.: Regularization of Newtonian functions via weak boundedness of maximal operators, to appear in J. Anal. Math

27. Royden, H.L.: Real Analysis, 3rd edn. Macmillan, New York (1988)

28. Shanmugalingam, N.: Newtonian spaces: An extension of Sobolev spaces to metric measure spaces. Rev. Mat. Iberoam 16, 243-279 (2000)

29. Shanmugalingam, N.: Harmonic functions on metric spaces. Illinois J. Math. 45, 1021-1050 (2001) 